\title{
Index
}

Abbott, Diane 121ff, 137

African Americans 20, 26, 27, 114 census counts 17

elected officials, non-partisan roles 116

employment 55-7, 58ff, 59-61;

female $57-8,61,70,125$;

male 56, 60, 62, 64-5, 67, 69-70, 125, 140ff

employment penalty, females 61 ghettos $25,31 \mathrm{ff}, 42,47-8,51-2$, $89,92,126,140$

health and mortality 50-1, 140

improved political opportunities $120,121-8,131-4$

inequality among 125

life chances 12,125

majority-minority politics 121

outmarriage 48-9

political class, creation of 53 , 121

political representation, comparisons with British minorities 116-17, 120-4 segregation 27-9, 39-42, 44, $46-8,50-1,140$

South-north

migration/integration 34-6

voting 118-19, 126

Afryie, Adam 137-8
Alba, R.D. 24

Al Qaeda 143

American Community Survey 13ff, 101

Amish community 43

Anderson, C.J. 74

anti-discrimination law 17,127 , 142

Arabs in the US 15

Asians in Britain

census questions $15 \mathrm{ff}$

immigration anxiety 108

inter-marriage 133

meaning of term 9, 12

segregation $38,107-8$

voting registration 118

Asians in the US

black politicians, electoral distribution 120

census 17

employment, South Asian males 64

ethnic diversity $13,124-5$

modern migration 16,37

outmarriage 48

segregation $36,38,46$

self identity 125

assimilation $22-4,32-53$

asylum seekers 20ff, 94, 99

Australia 14, 21

Australians 19, 23 
Bangladeshis in Britain 15ff, 53, 141

comparison with US

Bangladeshis 58, 62

employment 55, 61-2, 65-6,

71 ; female 57-8; male 56, 58,

$64-5,67$

employment penalty 61

population growth 10-11

racial threat 110,140

region of origin - British

migrants 38

relative life chances in Britain

18-19

segregation $38-9,42-3 \mathrm{ff}, 49$,

$51-3,140$

voluntary $v s$ involuntary

segregation 46

Bangladeshis in the US, employment premium 62

Belfast 42

Birmingham, Alabama 47

Birmingham, Britain 115, $120 \mathrm{ff}$

black Africans (Britain)

employment $64,66-7$

female employment 52

'black' and 'white', contested meanings of 9

black people (Britain and US) 12 analysis of 27

black consciousness 117

black immigrants to Britain 5, 12ff, 37

black immigrants to the US 37

black political class 53

black-white divide (colour line):

Britain 50; US 17-18, 37, 61

census: Britain 15ff; US 16, 17

co-residence with whites, US 28

election of black president

$112-21,128,144-5$

incarceration $50 \mathrm{ff}$

life chances 18 mixed race, US 17

possibility of black leader,

Britain 112-21, 135-8, 140-1

segregation from whites, US

44-6

white on black trust 76

Boateng, Paul 137

bonding within ethnic groups

85-91, 143

Booker, Cory 123

Boston, Mass. 37ff, 47

boundaries

ethnic 33, 35, 144

political 33, 35, 118-22

racial 47

Bradford 38-42, $120 \mathrm{ff}$

bridge-building between ethnic groups $84-7,90-1,143$

British Election Survey (2005)

$$
\text { 97-9 }
$$

British Empire and Commonwealth $5,11,17,139$

British Members of Parliament (MPs) 24

female 113

minority/majority seats $120 \mathrm{ff}$

minority members 116

party selection $136-7$

Brixton riots (1981 and 1985) 107

Broadwater Farm riot (1985) 107

Brown v. Board of Education 25

Burgess, S. 51

Canada 14, 43

Card, D. 51

Caribbeans (Britain) 107

census inclusion $15 \mathrm{ff}, 17$

comparisons with US

Caribbeans 37-40, 50

education: females 52-3; males 52-3

effect on attitudes towards immigration 108-10 
employment: all Carribeans 65-7; comparison with US Caribbeans 55-6, 64-5; female 57; male 58ff incarceration, relative incidence of 50

out-marriage 49,141

population increase $10 \mathrm{ff}$ segregation 17, 37-40, 53, 107, 119, 141; comparison with US 41

test scores (school) 51

Carribeans (US) education 49 incarceration, relative incidence of 50

racial threat $107-8$ segregation, comparison with Britain 41

Carter, Jimmy 8, 23

Catholic Church 19

Catholics 42, 119, 141

census data $5,19,27,30,36,38$, $40,44,46,48,50 \mathrm{ff}, 76,101$ 2000 census, US $3,15 \mathrm{ff}, 55$

2001 census, Britain 3, 11, 55, 121

2011 census, Britain 15, 36 relevant questions, US census $15-16,18,125$

US-British census differences 20 variation in ethnicity questions over time $9,11,18,39$

Charles, C.Z. $45-6$

Chicago 32-8, 40-2

Chicago School of Sociology 32, 35

Chinese (Britain) 84, 143 employment: males 55, 61, 64, 67; females with tertiary education 52, 53

population increase 10

relative importance as migrants 21
Chinese (US) 22, 139

employment: males 55, 62, 64, 66,67 ; females with tertiary education $52-3$

outmarriage 49

relative importance as migrants 21

chinatowns 33

'churning' of population $83-4$

citizenship 17, 24, 22, 140

Citizenship Survey of England and Wales (2005) 75-6

civil rights movement $16-17,114$, $117,136,145$ era $122,123,131$

clustering, ethnic $29,32,43,46$, $48,53,88,107$

co-ethnic identity $87 \mathrm{ff}$

cohort effects 131-2, 134ff

colonialism 5, 11

'color line' 16-17, 37, 124

Commission on Integration and Cohesion 23

community participation and community-mindedness 74 , 76-92

conflict theory $72-5,100$

Conservative Party 113-14

'A List' candidates 136-7

average member age, 1990s 136

black MPs 144

immigration as election issue 93, 99

leader election 136

contact theory 72-3, 75

councillors from ethnic minorities

$$
115-16
$$

'credit crunch' (2008) 20, 73

'cricket test' 23

crime rates 50,79, 80, 81

Crosby, Lynton 99

culture and cultural differences 24 , $38,49,58,72-3,117,119$, 139, 142 
Davis, Artur 123

Dawson, Michael 126

Democratic Party (Democrats)

112,126

Detroit 44-5

Dhanda, Parmjit 137

discrimination $16-17,47,55,119$

Du Bois, W.E.B. 16

Dylan, Bob 70

education and educational attainment 27, 47, 52-3, 113

African Americans 113, 117-18

British Muslims 140

differences between minority groups 50

education and employment differences 58-60, 65-6, 71

election issue, Britain 99

faith schools 144

immigration anxiety 101 , 104-6

segregation 47

social capital $79-80$

electoral systems and electoral laws 119, 121, 136-7

Empire Windrush 5

employment status (employed/ unemployed) 56-64;

see also 'salariat' jobs

enclaves, ethnic 26, 32, 37, 40, $42-3,90,92,107,143$

English language 143

ethnic 'penalty' and 'premium' 59-62, 68-71, 141

European Union 11, 22

Fair Housing Act (US, 1968) 25

faith schools 144

Federal Housing Association 47

First World War 34

Ford, R.G. 33

France 21, 24, 111
Gallup polls 45ff, 73ff, 94-5ff, 107-8, 127-30, 128ff

generational change $113,130-4,145$

Germany 14, 21, 22

ghettos and ghettoisation 6, 25-33

Afro-American preference to

leave 44-5, 140

British Muslims 140

discouragement of trust 89

educational achievement 51

English and US comparisons 29, 37-43, 51, 84-5, 89, 92, 140, 143

involuntariness $44-8$

majority-minority politics (US)

122

mobility from $32,125-6$

public concerns, Britain and US

29, 37-8, 90

radicalised politics (US) 122

Gingrich, Newt 16

Goodhart, David 72, 74, 81, 91

Grant, Bernie 137

Great Society programme 48

Hall, P. 74

Hauser, Philip 34

health standards $6,50-1,53,141$

Heath, A. $62 \mathrm{ff}, 74$

Hispanics (US) 12, 22, 128ff

ethnic classification for immigrants 22

education, women 52-3

employment 66,68 ; female 57 ;

male 55-6, 64-5, 67

employment penalties $62,66-8$

ethnic diversity in the US 13 ,

$16,20,124$

immigration anxiety 100

outmarriage 48-9

residential preferences $46-9$

resident in white minority

electoral districts $120-1$ 
segregation $36-8$

support for African American

candidates 120,126

support for Obama 125

US census question 15-16

whites' marriage attitudes

towards 133

housing schemes 47,73

Howard, John 99

Howard, Michael 99

Hurricane Katrina 25

Ifill, Gwen 123

immigration

anxiety about (Britain) 101, 104-6

controls on $12,16-17,34$

immigrants

employment penalties 61 , 68-70, 141

employment premium 62; women (Britain) 70

first-generation, employment factors 71

health: Britain 50; US 50

health premium/penalty 50, 141

incarceration 50

imprisonment 49-50, 141

Index of Dissimilarity (ID) 30-3,

$$
\text { 35-40, } 43
$$

Indians in Britain 107

education: performance 53; and women's employment 52-3, 57

education premium, males 66

employment 55,71 ; females 57 ;

males 56, 64-7, 141

employment penalties 61-2

enclaves 51

integration 84, 139

major regions of origin 38

population trends 10

promotion premium 66

segregation $40-2,53,107$
Indians in the US

employment, males $64-5,67$

employment premiums 62

tertiary education, women

52-3

inequality, social and economic

51, 79, 79ff, 113, 125, 142

institutional factors $47-8,112$,

$114,136,138$

integration

Afro Americans (US) 36, 45-6, 48,53

equality and diversity 23-4

government removal of barriers

143

inequality as a barrier to 142

measurement 31-2

minorities in Britain 49, 53

minority attitudes towards

46

schools (US) 134

trend towards (US) 36-7

US Army 92

In the Ghetto 25

Irish in Britain 9, 11, 107

census 15ff, 18-19ff

immigration anxiety 108-9

institutionalised discrimination 47

Irish in the US $12,21,70$

integration 139

Islam 18-19, 73, 140; see also

Muslims

Islamism 23

Italians in the US

integration 139, 143

segregation 32 , $37 \mathrm{ff}$

Italy 14

item response theory (IRT) 77

Jenkins, Roy 23

Jews in Britain 10

segregation $43 \mathrm{ff}$ 
Jews in the US

attitudes to marriage with 133

census 18

integration 139

Jewish humour and US identity 42 support for Jewish president 128

Johnson, Lyndon 48

Joshua 113, 121, 125, 137

Joshua generation 113

Kelly, Ruth 23

Key, V.O. 100

Keynes, J.M. 22

King, Martin Luther 25

Kipling, Rudyard 142

\section{Labour Party}

election of leaders 136

female representation 114

non-white representation 115 , 137

supporters 106, 108, 109-10

language

barriers for migrants 61,68 , 143

and homophily 142

views on minority languages 26

Le Pen, Jean Marie 111

Leicester 38, 40-2, 119-20

Ley, D. 44

life chances $18,43,65,125$

life expectancy 50

difference between males and females 51

'Little Sicilies' 32

Livingstone, Ken 26ff

London 2

Afro-Caribbean riots 107

Bangaldeshis in 46

Caribbeans in 39-40

Jewish in $43 \mathrm{ff}$

terrorist attacks on 18, 25, 110

Lublin, David 122ff, $125 \mathrm{ff}$ marriage patterns $48-9,92,141$

mixed-race $1,49,126-34,145$

media reporting and influence 6 , 93-7, 100, 103, 107, 110-11, 144

'melting pot' metaphor 9, 23-7, 32-4, 37-9, 49, 143

Mexican immigrants (US) 53

employment: female 57;

male 56, 64-7

employment penalty 55,62 , 68,70

illegal migrants $22 \mathrm{ff}$

relative importance among minorities 20-1

social capital 68,144

tertiary edcation, females 52

Mexico 21

Miami 38, 40-2

minorities

in Britain 27

in the US 27

mixed-race individuals 17,49

mixed-race marriage 126-34, 145

Moses 113, 122

Moses generation 111, 113, 122,137

multiculturalism 18-19

Muslims 22ff, 43, 73ff, 141-2

anti-Muslim attitudes 110

communities 38, 110, 140-2

dress 18-19, 143

faith schools 144

integration (Britain) 110

population (US) 19

socio-economic status 19, 140

see also Islam

nationalism, British 4

Native Americans 12-13, 16

Nee, V. 24

New Orleans 25 
New York 12, 37, 39-40, 121

Northern Ireland 2ff, 42

Obama, Barack

comparison with Thatcher 114 factors of victory 112-21, 128,

144-5

imitation of Obama victory

112-21, 135-8, 140-1

personal background 1, 5

significance of presidency 6-8, 53

O'Connor, Sandra Day 121

'one drop' rule 17

opinion polls $45 \mathrm{ff}, 94-5,97,101$, $103,107,113 \mathrm{ff}, 118 \mathrm{ff}, 127 \mathrm{ff}$, 134-5, 145

Ouseley, Herman 26

Pakistanis in Britain assimilation comparison to African Americans 41

census 15

education 66

employment 55, 62, 71; female

53 , 58; male 53, 61-2, 64-5, 67

employment penalty 66

employment status comparison with Indians 65, 141

health status 51

population 10

racial threat 110

region of origin 38

relative school test scores 51

segregation 38, 40-2, 51-2, 107

social status $19,38,53,140$

violence against 107

Pakistanis in the US

employment: female 58; male 64

employment premium 62

Palin, Sarah 93
Park, Robert 32

Paskeviciute, A. 74

Patrick, Deval 118, 123

Pennant, R. 74

Pew Center 19, 22ff, 94ff, 101, 103

Philadelphia 44

Phillips, Trevor

claims of ethnic ghettoisation 25-6, 29, 37, 42

obstacles for minorities in

Britain 112-13, 136

Philpott, Thomas 32

Polish immigrants integration in Chicago, US 32-5 migration to Britain 11, 20

political correctness 93, 130, 145 politicians from ethnic minorities (Britain and the US) 115-38, 145

post-civil rights era 125

poverty 6,44

calculation $79 \mathrm{ff}$

as a factor in segregation 46,51

inequality among Afro-

Americans 125, 82-4, 91

negative factor in community mindedness for whites in

Britain 81-4, 91

Powell, Enoch current relevance 111 ethnic segregation 107, 111 'foaming with blood' speech (1968) 99-100

prejudice, racial as basic human emotion $126-7$ and cohort effects 131, 134 in employment 55, 65 and political correctness 130

Presley, Elvis 25

public opinion and concern 6, $111,136,138$

causal relationship with media activity $95-6$ 
on migrant groups $107-10$

mismatch with reality 94

on mixed-race marriage $127-8$

Obama 144

political campaigns 97,103

situational factors on attitudes

to immigration $103-7$

volatility 94

Putnam, R.D. 75ff, 92ff, 136

social capital 74

social diversity and community

mindedness 91

trust 75

race

conceptual issues 8-9

perceptions of 5

researching with migration $20-2$

surveying and policy: US and

Britain 15-18, 20, 22-4

race relations legislation 17

racism

elite opinion 6, 23

explaining marginalisation 65

institutionalised 47, 94

migration controls $17,71-2,94$, 111

separating cohort and

generational 130

religious differences $23-4$

integration $92,140-2$

measurement 18

minorities 19, 42

Republican Party 8ff, 93

'rippling waves' metaphor 32-3, 37

Roosevelt, Theodore 8, 23

Rothstein, J. 51

Saini, Pardeep 2

'salad bowl' metaphor 23-4, 27, 143

'salariat' jobs 63ff, 140

Schuman, H. 128, 134
Second World War 11ff, 92

segregation

British Muslims 140

gender 53

institutions $47-8$

integration 48-9

marginalisation $49-53$

measurement 30-1

political advantage 119

residential $24-5,118$

'sleepwalking into segregation'

88

US 27, 35-7

US and Britain 6, 26, 37-43

voluntary and enforced 43-6

Sen, Amartya 139, 142

slavery $4-5,16-17,20,140$

social capital

community health 73-9

diversity 78-92

measurement 77

US and Britain 75-84

Social Capital Community

Benchmark Study (2000) 75

Social Change: A Harvard-

Manchester initiative

(SCHMI) 1, 6

social distance $126-7,130,135$,

142-3

South Africa 44

Statue of Liberty 4

Straw, Jack 18-19

The Sun 94

support networks 43

Supreme Court, US 25

Taeuber, K.A. and A.F. 46

Taylor, John 137

Tebbit, Norman 23

terrorist attacks: on London

(July 2005) 18, 25, 110; on

Washington (September

2001) 95-6, 110-11 
Thatcher, Margaret 113-14, 138 tolerance 128-36, 145

Tomasky, Michael 124

Tower Hamlets 46 trust $74-5,81$

unemployment $55-6,70$

values 73

Vaz, Keith 137

Voltaire 72, 74

voting behaviour 118
Voting Rights Act (US, 1965) 114, 119

Wales 15

West Indians 15, 107; see also Caribbeans

Wittgenstein, Ludwig 130 women politicians 113-14

women's education $51-2$

women's employment $56-8,61$

xenophobia 144 